Pacific Journal of Mathematics

GREEN'S FUNCTION INEQUALITIES FOR TWO-POINT 


\title{
GREEN'S FUNCTION INEQUALITIES FOR TWO-POINT BOUNDARY VALUE PROBLEMS
}

\author{
P. W. Bates and G. B. Gustafson
}

Simple inequalities are obtained for the Green's function $G(t, s)$ of a two-point boundary value problem for a $k^{\text {th }}$ order linear ordinary differential equation. The constants appearing in the inequalities are best possible. Each estimate has right side a product of a function of $t$ and a function of $s$. Illustrations are given for $k=2,3,4$ and certain $k^{\text {th }}$ order constant coefficient operators.

1. Introduction. The purpose of this paper is to obtain a separable inequality of the form

$$
|G(t, s)| \leqq \sum_{i=1}^{2} p_{i}(t) q_{i}(s) \chi_{E_{i}}(t, s),(t, s) \in[a, b] \times[a, b],
$$

for the Green's function $G(t, s)$ of the two-point boundary value problem

$$
K v=f(t), v^{(i)}(a)=v^{(j)}(b)=0,0 \leqq i \leqq l-1,0 \leqq j \leqq k-l-1 .
$$

The linear ordinary differential operator $K$ has coefficients smooth enough to define its adjoint operator $K^{*}$, the operator $K$ is assumed disconjugate on $[a, b]$ (see Section 2 ), $E_{1} \cup E_{2}=[a, b] \times[a, b], E_{1} \cap E_{2}=$ $\phi$, and $f \in C[a, b]$. For non-smooth coefficients, see Section 6 .

The motivation for this kind of inequality is in the study of the linear integral operator

$$
T f=\int_{a}^{b} G(t, s) f(s) d s
$$

associated with problem (1.2). Indeeed, (1.1) provides a practical estimate for $\|T\|_{X}$ in various Banach spaces $X$, and allows very tight manipulations with the integral equation $v=T f$.

The demands that we make on (1.1) is that it be an identity for $k=2$, and that it cannot be improved for $k \geqq 3$, in the sense that

$$
\sup _{(t, s) \in E_{i}}\left|\frac{G(t, s)}{p_{i}(t) q_{i}(s)}\right|=1 \quad(i=1,2) .
$$

The main result is Theorem 3.1, in which we prove the two-point inequality

$$
|G(t, s)| \leqq \min \left\{\frac{v(t) v^{*}(s)}{v^{(l)}(a)}, \frac{w(t) w^{*}(s)}{\left|w^{(k-l)}(b)\right|}\right\}
$$


This takes the form of (1.1) for appropriate definition of $E_{1}$ and $E_{2}$. The functions are positive on $(a, b)$ and satisfy $K v=K w=K^{*} v^{*}=$ $K^{*} w^{*}=0$, plus certain boundary conditions at $a$ and $b$ [see (3.1) and (3.2)].

The proof of (1.3) appears in $\S 3$, with suitable preparation being done in $\S 2$.

Illustrations of inequality (1.3) appear in $\$ \S 4$ and 5, and applications are discussed in $\S 7$. In particular, we obtain the Ostroumov inequality [9] for $K \equiv(d / d t)^{k}$ (see (4.2) infra).

A general discussion of constant coefficient operators appears in $\S 5$. Illustrations are given to show the nonspecialist how to find the interval of disconjugacy and the functions $v, v^{*}, w, w^{*}$ in (1.3).

2. Preliminaries. Consider the linear ordinary differential equation

$$
K v=0 ; K v \equiv v^{(k)}+\sum_{i=0}^{k-1} p_{i}(t) v^{(i)}
$$

The adjoint equation is defined by

$$
K^{*} u=0 ; K^{*} u \equiv(-1)^{k} u^{(k)}+\sum_{i=0}^{k-1}(-1)^{1}\left(p_{i} u\right)^{(i)} .
$$

Throughout the paper, $p_{i} \in C^{i}[a, b]$, whenever $K^{*}$ is defined.

The two-point boundary-value problem

$$
K u=f ; u^{(i)}(a)=u^{(j)}(b)=0,0 \leqq i \leqq l-1,0 \leqq j \leqq k-l-1 .
$$

is assumed to have a Green's function $G(t, s)$, so that

$$
u(t)=\int_{a}^{b} G(t, s) f(s) d s .
$$

Let us make the stronger hypothesis that $K$ is a disconjugate operator on $[a, b]$. This means that the only solution of $K u=0$ with $k$ zeros, counting multiplicities, is $u \equiv 0$. Then $K^{*}$ is also disconjugate; see Sherman [10].

The hypothesis of disconjugacy on $[a, b]$ is known to be equivalent to the factorization of $K$ on $[a, b]$ into first-order operators:

$$
K u=b_{k}^{-1}\left(\cdots\left(b_{1}^{-1}\left(b_{0}^{-1} u\right)^{\prime}\right)^{\prime} \cdots\right)^{\prime} .
$$

The functions $b_{i}$ are positive on $[a, b]$. This is called the LibriFrobenius-Mammana factorization; see Willett [11], Libri [8].

Let $\left\{u_{m n}^{*}(t)\right\}$ be the set of solutions of $K u=0$ satisfying $u_{0, j}^{*(p)}(b)=$ $u_{1, i}^{*(q)}(a)=0, u_{0, j}^{*(q)}(a)=\delta_{j q}, u_{1, i}^{*(p)}(b)=\delta_{i p}, 0 \leqq j, q \leqq l-1,0 \leqq i, p \leqq$ $k-l-1$, and put 


$$
U(t)=\left[u_{0,0}^{*}(t), \cdots, u_{0, l-1}^{*}(t), u_{1,0}^{*}(t), \cdots, u_{1, k-l-1}^{*}(t)\right] .
$$

Let $W(t)$ be the Wronskian matrix generated by $U(t)$, and let $V(s)=\operatorname{diag}\left[\chi_{E_{0}}(s), \cdots, \chi_{E_{0}}(s), \chi_{E_{1}}(s), \cdots, \chi_{E_{1}}(s)\right]$, with $l$ spaces occupied by $\chi_{E_{0}}(s), E_{0}=\{a\}, E_{1}=[a, b], V(s)$ being $k \times k$.

Put $h(t, s)=U(t) W^{-1}(s) e, e=(0, \cdots 0,1)^{T}$. The function $h(t, s)$ is the solution of $K u=0$ satisfying $h^{(j)}(s, s)=\delta_{j, k-1}($ Cauchy function).

The Green's function $G(t, s)$ for problem (2.3) is given by (see Gustafson [5]) the vector-matrix identity

$$
G(t, s)=U(t)[\varepsilon(t-s) I-V(s)] W^{-1}(s) e
$$

where $\varepsilon(t-s)=1$ for $t-s>0, \varepsilon(t-s)=0$ otherwise. The form of (2.4) to be used most often is the scalar identity

$$
G(t, s)=\left\{\begin{array}{cc}
\sum_{j=0}^{l-1} u_{0, j}^{*}(t) h^{(j)}(a, s), & s \leqq t, \\
-\sum_{j=0}^{k-l-1} u_{1, j}^{*}(t) h^{(j)}(b, s), & t \leqq s .
\end{array}\right.
$$

The following lemmas are singled out for later use. The first, on continuity of $(t, s) \rightarrow G(t, s)$, does not require that $p_{i} \in C^{i}[a, b]$, nor is disconjugacy needed; it follows immediately from (2.5). The second is a consequence of the Peano identities (Hartman [7], p. 95).

LEMmA 2.1. The Green's function $G(t, s)$ for the two-point boundary value problem (2.3) is continuous on the square $[a, b] \times[a, b]$.

LEMmA 2.2. Let $h(t, s)$ be the solution of $K u=0, u^{(i)}(s)=\delta_{i, k=1}$. Assume that $K^{*}$ is defined, then:

$$
K^{*}\left[h^{(j)}\left(t_{0}, s\right)\right]=0 \text { for each fixed } t_{0} \text {. }
$$

$$
\begin{gathered}
{\left[\frac{\partial}{\partial s}\right]^{i} h^{(j)}(x, x)=0 \text { for } i+j<k-1,} \\
{\left[\frac{\partial}{\partial s}\right]^{i} h^{(k-1-i)}(x, x)=(-1)^{i} .}
\end{gathered}
$$

\section{LEMMA 2.3. Let}

$$
\begin{aligned}
& \varphi_{p}(s)=\sum_{j=0}^{k-l-1} u_{1, j}^{*(p)}(a) h^{(j)}(b, s), \\
& \psi_{r}(s)=\sum_{j=0}^{l-1} u_{0, j}^{*(r)}(b) h^{(j)}(a, s) .
\end{aligned}
$$

Then $\varphi_{p}$ has $k-p-1$ zeros at $a, l \leqq p \leqq k-1$, and $\psi_{r}$ has $k-r-1$ zeros at $b, k-l \leqq r \leqq k-1$. 
Proof. First, we record that $h(t, s)=U(t) W^{-1}(s) e$.

After a short calculation with the basis $U=\left(u_{0,0}^{*}, \cdots, u_{1, k-l-1}^{*}\right)$, one finds that

$$
\psi_{r}(s)=\frac{\operatorname{det} \Phi_{0}(s)}{\operatorname{det} W(s)}
$$

where $\Phi_{0}(s)$ is the matrix with successive rows $U(s), \cdots, U^{(k-2)}(s)$ and last row $\left(u_{0,0}^{*(r)}(b), \cdots, u_{0, l-1}^{*(r)}(b), 0, \cdots, 0\right)$.

Differentiating this relation and using the Liouville identity [7] we obtain

$$
\psi_{r}^{\prime}(s)=\frac{\operatorname{det} \Phi_{1}(s)}{\operatorname{det} W(s)}-p_{k-1}(s) \psi_{r}(s)
$$

where $\Phi_{1}(s)$ is the same as $\Phi_{0}(s)$, except we must replace $U^{(k-2)}(s)$ by $U^{(k-1)}(s)$. By induction,

$$
\psi_{r}^{(\alpha)}(s)=\frac{\operatorname{det} \Phi_{\alpha}(s)}{\operatorname{det} W(s)}+\sum_{i=0}^{\alpha-1} f_{i, \alpha}(s) \psi_{r}^{(i)}(s)
$$

where $f_{i, \alpha}$ has $k-\alpha+i$ continuous derivatives, and $\Phi_{\alpha}(s)$ has the same last row as $\Phi_{0}(s)$, the same first $k-\alpha-1$ rows as $\Phi_{0}(s)$, but the next rows are $U^{(k-\alpha)}(s), \cdots, U^{(k-1)}(s)$.

For $0 \leqq \alpha \leqq k-r-2$ each $\Phi_{\alpha}(b)$ has the block matrix form

$$
\left[\frac{0}{E} \mid \frac{I}{F}\right]
$$

where $E$ has two equal rows, $I=(k-l) \times(k-l)$ identity. Therefore, $\operatorname{det} \Phi_{\alpha}(b)=0$. This proves that $\psi_{r}$ has $k-r-1$ zeros at $b, k-l \leqq$ $r \leqq k-1$.

The proof for $\varphi_{p}$ is similar, and will be omitted.

REMARK. In the notation of Gustafson [5], the function $\varphi_{p}$ has a zero of order $(k-p-1, l)$ at $\{a, b\}$, and $\psi_{r}$ has a zero of order $(k-l, k-r-1)$. These functions collectively form a basis for $K^{*} u=0$.

3. The Green's function inequality. Let $v(t), v^{*}(s), w(t), w^{*}(t)$, be defined by the following relations:

(3.1) $K v=K^{*} v^{*}=0, v$ and $v^{*}$ have zeros of order $(l, k-l-1)$ and $(k-l-1, l)$ at $\{a, b\}$, resp., with $v^{(k-l-1)}(b)=(-1)^{k-l-1}$ and $v^{*^{(k-l-1)}}(a)=1$.

(3.2) $K w=K^{*} w^{*}=0, w$ and have zeros of order $(l-1, k-l)$ 
and $(k-l, l-1)$ at $\{a, b\}$, resp., with $w^{(l-1)}(a)=1, w^{*(l-1)}(b)=(-1)^{l-1}$.

The functions defined in (3.1), (3.2) are positive on $(a, b)$ because of the disconjugacy of the operators $K$ and $K^{*}$.

\section{Two-point Inequality Theorem}

THEOREM 3.1. Let $p_{i} \in C^{i}[a, b](0 \leqq i \leqq k-1)$ and assume $K$ is disconjugate on $[a, b], v, v^{*}, w, w^{*}$ are given by (3.1), (3.2). Then the Green's function $G(t, s)$ for problem (2.3) satisfies the inequality

$$
|G(t, s)| \leqq \min \left\{\frac{1}{v^{(l)}(a)} v(t) v^{*}(s), \frac{1}{\left|w^{(k-l)}(b)\right|} w(t) w^{*}(s)\right\}
$$

for $a \leqq t, s \leqq b$. The constants are best possible.

CoRollaRY 3.2. On the upper triangle $a \leqq t \leqq s \leqq b$,

$$
|G(t, s)| \leqq \frac{1}{v^{(l)}(a)} v(t) v^{*}(s)
$$

and on the lower triangle $a \leqq s \leqq t \leqq b$,

$$
|G(t, s)| \leqq \frac{1}{\left|w^{(k-l)}(b)\right|} w(t) w^{*}(s) .
$$

Both (3.3) and (3.3) $)_{\mathrm{c}}$ hold on the entire square $[a, b] \times[a, b]$. Inequality (3.3) $)_{\mathrm{a}}$ is of the correct order of magnitude, in the sense that $|G(t, s)| /\left[\right.$ The RHS of $\left.(3.3)_{\mathrm{a}}\right] \rightarrow 1$ as $(t, s) \rightarrow \partial([a, b] \times[a, b])$; see Lemmas 3.2, 3.3, 3.4, infra.

Proof of Theorem 3.1. The proof of (3.3) ${ }_{\mathrm{c}}$ on $[a, b] \times[a, b]$ follows in the same way as (3.3), so it will be omitted. For convenience, let us put $u(s)=v^{*}(s)$ hereafter.

The theorem will be proved by the sequence of lemmas to follow. To introduce notation, let

$$
k(t, s)=\frac{G(t, s)}{v(t) u(s)}, a<t, s<b .
$$

Then inequality (3.3) ${ }_{b}$ on the open square is the same as $(-1)^{k-l} k(t, s) \leqq$ $1 / v^{(l)}(a)$. Here we have used the fact that $(t-a)^{l}(t-b)^{k-l} G(t, s)>0$ on the open square (see Coppel [3], p. 108).

For $k=2$, one can easily verify (3.3), because in this case

$$
G(t, s)=\left\{\begin{array}{l}
-\frac{v(t) v^{*}(s)}{v^{\prime}(a)}, t \leqq s, \\
-\frac{v(s) v^{*}(t)}{v^{\prime}(a)}, s \leqq t .
\end{array}\right.
$$


The inequalities (3.3) hold on the entire square because $w(t)=v^{*}(t)$, $w^{*}(t)=v(t)$, and $v(x) v^{*}(y) \leqq v(y) v^{*}(x)$ for $x \leqq y$.

For $k \geqq 3$, the theorem follows from (3.4), (3.9), (3.11), (3.13).

The problem with the function $k(t, s)$ given in (3.4) is that it is not defined on the boundary of the square. If one examines the Green's function for the $(2,1)$-problem for $K=[d / d t]^{3}$ :

$$
G(t, s)=\left\{\begin{array}{l}
{\left[\frac{b-t}{b-a}+\frac{t-a}{b-a} \frac{b-t}{b-a}\right] \frac{(a-s)^{2}}{2}} \\
\quad+\frac{(t-a)(b-t)(a-s)}{b-a}, s \leqq t, \\
-\left[\frac{t-a}{b-a}\right]^{2} \frac{(b-s)^{2}}{2}, t \leqq s,
\end{array}\right.
$$

then we seen that $k(t, s)$ has a jump discontinuity at $(a, a)$ and $(b, b)$. In fact, on the upper triangle, $k(t, s) \sim(b-a) / 2$ near $(a, a)$ and $(b, b)$, but on the lower triangle $k(t, s) \sim 0$.

Most of our work below is aimed at settling upon the correct boundary values for $k(t, s)$. It turns out that $k(t, s)$ will assume only two values on the boundary. Then we show that sup $|k|$ must occur on the boundary, and write down the answer. The reader will find (3.6) useful for interpretation of the various arguments to follow.

Lemma 3.2. The quotient $k(t, s)$ can be defined on the upper triangle $\mathscr{T}_{u}=\{(t, s): t \leqq s\}$ so as to have the following properties:

$$
\begin{gathered}
k(t, s) \text { is bounded on } \mathscr{T}_{u} \\
k(t, s) \text { is continuous on } \mathscr{T}_{u} \backslash\{(a, a),(b, b)\} \\
k(t, s)=\frac{(-1)^{k}}{u^{(l)}(b)} \text { on } \Omega_{u}=\{(t, s): t=a \text { or } s=b\} .
\end{gathered}
$$

Proof. Define $k(t, s)$ on $\Omega_{u}$ by relation (3.9). It will be shown that (3.8) is valid. We use below the Landau symbol " $O$ ", defined by $f(t)=O(g(t))$ iff for some $M>0,|f(t)| \leqq M|g(t)|$ as $t \rightarrow \omega$.

The first step of the proof is to assemble with the help of (2.7), (2.8), (3.1), (3.2) the following order relations:

$$
\begin{aligned}
h^{(j)}(b, s) & =O\left(|s-b|^{k-j-1}\right)(0 \leqq j \leqq k-1), h^{(k-l-1)}(b, s) \\
& =(b-s)^{l}+O\left(|b-s|^{l+1}\right),[u(s)]^{-1} \\
& =O\left[|s-b|^{-l}\right] \text { as } s \rightarrow b ; \\
{[u(s)]^{-1} } & =O\left(|s-a|^{-k+l+1}\right) \text { as } s \rightarrow a ; \\
{[v(t)]^{-1} } & =O\left(|t-a|^{-l}\right) \text { as } t \rightarrow a ;
\end{aligned}
$$




$$
\begin{aligned}
& {[v(t)]^{-1}=0\left(|t-b|^{-k+l+1}\right) \text { as } t \rightarrow b ;} \\
& u_{1, j}^{*}(t)=0\left(|t-b|^{j}\right) \text { as } t \rightarrow b ; \\
& u_{1, k-l-1}^{*}(t)=(-1)^{k-l-1} v(t) .
\end{aligned}
$$

To analyze the boundary behavior of $k(t, s)$, write $k(t, s)=$ $-\sum_{j=0}^{k \rightarrow l-1} Q_{j}(t, s)$, where $Q_{j}(t, s) \equiv u_{1, j}^{*}(t) h^{(j)}(b, s) /[u(s) v(t)]$, using relation (2.5).

Since $|b-s| \leqq|b-t|$ for $(t, s) \in \mathscr{T}_{u}$, it follows that $Q_{k-l-1}(t, s) \rightarrow$ $(-1)^{k} / u^{(l)}(b)$ and $Q_{j}(t, s) \rightarrow 0(0 \leqq j \leqq k-l-2)$ as $(t, s) \rightarrow(a, b)$. Therefore (3.8) is correct at $(a, b)$.

By virtue of Lemma 2.2 , one can write $k(t, s)=(-1)^{k} / u^{(l)}(b)+$ $0(|s-b|)$ uniformly on compact subsets of $a<t<b$ as $s \rightarrow b$. Therefore, (3.8) is correct along the upper edge of $\mathscr{T}_{u}$.

To verify (3.8) along the left edge of $\mathscr{T}_{u}$, each $u_{1, j}^{*}(t)$ is replaced by its Taylor expansion about $t=a$ in the expression $Q_{j}(t, s)$, and an application of Lemma 2.3 gives $k(t, s)=-\varphi_{l}(s) /\left[u(s) v^{(l)}(a)\right]+0(|t-a|)$ as $t \rightarrow a$, uniformly on compact subsets of $a<s<b$.

The functions $\varphi_{l}$ and $u$ have the same zero properties at $s=a$ and $s=b$, therefore by the disconjugacy assumption they must be constant multiples of one another. Using Lemmas 2.2, 2.3 and Green's formula (Hartman [7], p. 67) one can establish the following identities: $\varphi_{l}(s)=(-1)^{k-l-1} u(s) ;(-1)^{l} v^{(l)}(a)=u^{(l)}(b)$. Consequently,

$$
k(t, s)=(-1)^{k} / u^{(l)}(b)+0(|t-a|) \text { as } t \longrightarrow a,
$$

uniformly on compact subsets of $a<s<b$. This completes the proof of (3.8).

To verify (3.7), one only needs to examine small neighborhoods of $(a, a)$ and $(b, b)$. The details are left to the reader.

LEMmA 3.3. The quotient $k(t, s)$ can be defined on the lower triangle $\mathscr{T}_{l}=\{(t, s): t>s\}$ to have the following properties

$$
k(t, s) \text { is bounded on } \mathscr{T}_{l} \text {. }
$$

$$
\begin{gathered}
k(t, s)=0 \quad \text { on } \quad \Omega_{l}=\left\{(t, s) \in \mathscr{T}_{l}: t=b \quad \text { or } s=a\right\} . \\
k(t, s) \quad i s \text { continuous on } \mathscr{T}_{l} .
\end{gathered}
$$

Proof. The proof proceeds in the same way as Lemma 3.2, with appropriate use of Lemmas 2.2 and 2.3. The details are left to the reader.

Lemma 3.4. Let $k(t, s)$ be defined on $\Omega=[a, b] \times[a, b]$ as in Lemmas 3.2, 3.3. Then 


$$
\sup _{\Omega}|k(t, s)|=\sup _{\partial \Omega}|k(t, s)|=\frac{(-1)^{l}}{u^{(l)}(b)}=\frac{1}{v^{(l)}(a)} .
$$

Proof. Suppose not, then there exists $\left(t_{0}, s_{0}\right) \in \Omega$ such that $\left|k\left(t_{0}, s_{0}\right)\right|>\sup _{\partial \Omega}|k(t, s)|$. Therefore, $\left(t_{0}, s_{0}\right)$ must belong to the interior of $\Omega$. Consider the function $y(t)=k\left(t, s_{0}\right), a \leqq t \leqq b$. This function is continuous on $a \leqq t \leqq b$, by Lemmas 2.1,3.2, 3.3, and differentiable on $a<t<b$.

The derivative $y^{\prime}(t)$ on $a<t<b$ cannot vanish. Indeed, suppose $y^{\prime}\left(t_{1}\right)=0$ for some $t_{1} \in(a, b)$. Then

$$
0=y^{\prime}\left(t_{1}\right)=\frac{W\left(v(t), G\left(t, s_{0}\right)\right)}{v^{2}(t) u\left(s_{0}\right)} \text { at } t=t_{1},
$$

where $W$ is the Wronskian determinant. Define $\alpha=G\left(t_{1}, s_{0}\right) / v\left(t_{1}\right)$, then the function

$$
z(t) \equiv \alpha v(t)-G\left(t, s_{0}\right)
$$

has a double zero at $t=t_{1} \in(a, b)$. The function $z(t)$ has a zero of order $l$ at $t=a, k-l-1$ at $t=b$, hence $k+1$ zeros in $[a, b]$. However, the function $z(t)$ is a solution of the disconjugate equation $K z=0$ on $a \leqq t \leqq s_{0}$ and on $s_{0} \leqq t \leqq b$. By the proof of Theorem 11 in Coppel [3], p. 108, it follows that $z(t) \equiv 0, a \leqq t \leqq b$. Therefore,

$$
\frac{G\left(t, s_{0}\right)}{v(t)}=\alpha, a \leqq t \leqq b .
$$

Letting $t \rightarrow b$ we find that $\alpha=0$, which is a contradiction.

This proves that $y(t)$ is monotonic on $[a, b]$. Since $y(a)=$ $(-1)^{k} / u^{(l)}(b), y(b)=0$, it follows that

$$
\left|k\left(t_{0}, s_{0}\right)\right| \leqq\left|k\left(\alpha, s_{0}\right)\right| \leqq \sup \partial \Omega|k(t, s)| .
$$

This contradicts our original assumption on $\left(t_{0}, s_{0}\right)$. Therefore, (3.13) holds, because the supremum over the boundary is the larger of 0 and $(-1)^{l} / u^{(l)}(b)$. The proof is complete.

REMARK 3.5. The inequality (3.3) ${ }_{\mathrm{b}}$ is the best result of its kind, because we may divide by $v(t) v^{*}(s)$ in $(3.3)_{\mathrm{b}}$ and let $(t, s) \rightarrow(a, b)$ to obtain equality. In $(k-1,1)$-problems, equality holds in $(3.3)_{\mathrm{b}}$ in the entire upper triangle. Similar statements hold for (3.3)c.

The monotonicity argument of Lemma 3.4 shows that $(3.3)_{a}$ is weakest along the diagonal $t=s$. For example, the behavior of (3.6) along $t=s$ is quite usual.

Inequalities of this same type hold for singular boundary-value problems. However, the application of the Green's function inversion 
method is more complicated, and is an integral part of the selection of the space of action of the integral operator. For example, the singular problem

$$
\begin{gathered}
y^{\prime \prime}=f(t), \\
y(0)=0, y(\infty)=0,
\end{gathered}
$$

under ordinary inversion loses the boundary value at $\infty$. A definitive statement about the options here will be the subject of a future paper.

4. The Ostroumov inequality for $D^{k}$. Consider the problem

$$
y^{(k)}=f(t), y^{(i)}(a)=y^{(j)}(b)=0,0 \leqq i \leqq l-1,0 \leqq j \leqq k-l-1,
$$

and denote by $G(t, s)$ the corresponding Green's function (see [5]). The Ostroumov inequality [9] can be obtained from (3.3)

$$
|G(t, s)| \leqq \frac{(t-a)^{l}(b-t)^{k-l-1}(s-a)^{k-l-1}(b-s)^{l}}{l !(k-l-1) !(b-a)^{k-1}} .
$$

It is only necessary to verify by inspection that

$$
v(t)=\frac{(t-a)^{l}(b-t)^{k-l-1}}{(k-l-1) !(b-a)^{l}}, v^{*}(s)=\frac{(s-a)^{k-l-1}(b-s)^{l}}{(k-l-1) !(b-a)^{l}}
$$

and then compute

$$
v^{(l)}(a)=\frac{l !(b-a)^{k-l-1}}{(k-l-1) !(b-a)^{l}},
$$

for then (4.2) follows from (3.3) $\mathrm{b},(4.3),(4.4)$.

However, inequality (3.3) $)_{\mathrm{a}}$ is a decided improvement over (4.2). One easily verifies that

$$
\frac{v(t) v^{*}(s)}{v^{(l)}(a)}=M(t, s) \frac{w(t) w^{*}(t)}{\left|w^{(k-l)}(b)\right|}
$$

where

$$
M(t, s)=\frac{k-l}{l} \frac{(t-a)(b-s)}{(s-a)(b-t)} .
$$

The level set $M(t, s)=1$ for $k=2 l$ is the diagonal $t=s$. For $k \neq$ $2 l$, the level set $M(t, s)=1$ is the curve

$$
(s-a)=\frac{(b-a)(l-k)}{2 l-k}+\frac{(b-a)^{2} l(l-k)}{(2 l-k)^{2}} \cdot \frac{1}{t-a-(b-a) l /[2 l-k]},
$$

which goes through $(a, a),(b, b)$ and is concave for $2 l-k>0$, convex 
for $2 l-k<0$.

If we make use of this curve, then we have

$$
\begin{aligned}
|G(t, s)| \leqq \frac{(t-a)^{l}(b-t)^{k-l-1}(s-a)^{k-l-1}(b-s)^{l}}{l !(k-l-1) !(b-a)^{k-1}} \chi_{E_{1}}(t, s) \\
\\
+\frac{(t-a)^{l-1}(b-t)^{k-l}(s-a)^{k-l}(b-s)^{l-1}}{(l-1) !(k-l) !(b-a)^{k-1}} \chi_{E_{2}}(t, s)
\end{aligned}
$$

where $\chi_{E}$ denotes the characteristic function of the set $E$ and $E_{1}=$ $\{(t, s): M(t, s) \leqq 1\}, E_{2}=\{(t, s): M(t, s)>1\}$.

This shows that $(3.3)_{\mathrm{b}}$ is better than $(3.3)_{\mathrm{c}}$ for $(t, s) \in E_{1}$, whereas the opposite is true for $(t, s) \in E_{2}$.

When $k=2 l, E_{1}$ is the upper triangle $t \leqq s$ and $E_{2}$ is the lower triangle $s<t$.

The advantage of this observation is that

$$
\begin{aligned}
\int_{a}^{b}|G(t, s)||f(s)| d s & \leqq \frac{v(t)}{v^{(l)}(a)} \int_{a}^{b} v^{*}(s)|f(s)| \chi_{E_{1}} d s \\
& +\frac{w(t)}{\left|w^{(k-l)}(b)\right|} \int_{a}^{b} w^{*}(s)|f(s)| \chi_{E_{2}} d s .
\end{aligned}
$$

Now you can use the definitions of $E_{1}$ and $E_{2}$ to make the right side the sum of two Volterra operators.

There are other inequalities in the literature which are similar to (4.2). The most notable is due to Beesack [1], and we refer the reader to this paper and the references therein.

5. Disconjugate operators with constant coefficients. The constant coefficient operator

$$
K v \equiv v^{(k)}+\sum_{i=0}^{k-1} a_{i} v^{(i)}
$$

will be disconjugate on an interval $[a, b]$ iff it is disconjugate on $[0$, $b-a]$, because the equation is autonomous.

Due to the theory of extremal solutions, carried out by Sherman [10], $K u=0$ will be disconjugate on $[0, T]$ iff the only solution $u$ with a zero of order $l$ at 0 , order $k-l$ at $T, u>0$ on $(0, T)$, is $u \equiv$ 0 , for $1 \leqq l \leqq k-1$. Together with linear algebra, this gives an effective way to test the disconjugacy of $K$. For example, if $K y=$ $y^{\prime \prime \prime}+y^{\prime}$, then it is relatively easy to show that $K$ is disconjugate on $[a, b]$ iff $b-a<2 \pi$, because any solution with a double zero at $c$ must be multiple of $1-\cos (t-c)$.

The adjoint operator

$$
K^{*} u=(-1)^{k} u^{(k)}+\sum_{i=0}^{k-1} a_{i}(-1)^{i} u^{(i)}
$$


also has constant coefficients, therefore the possibility of computing the various functions in inequality (3.3) is close at hand. Indeed, $v(-s)$ is a solution of $K^{*} u=0$, hence also $v(-s+c)$ is a solution for any real $c$. But $v(b+a-s)$ vanishes $k-l-1$ times at $a$ and $l$ times at $b$, therefore, in the case of constant coefficients,

$$
v^{*}(s)=v(b+a-s), w^{*}(s)=w(b+a-s) .
$$

On the other hand, one can write down $v(t)$ in terms of any standard basis $\left\{v_{1}, \cdots, v_{k}\right\}$ for $K v=0$, as follows. Let $V(t)=\left(v_{1}(t)\right.$, $\left.\cdots, v_{k}(t)\right)$, then let $\Phi_{l}(t ; T)$ be the matrix with rows $V(0), V^{\prime}(0), \cdots$, $V^{(l-1)}(0), V(T), V^{\prime}(T), \cdots, V^{(k-l-2)}(T), V(t)$. Let $\Psi_{l}(T)$ be the same as $\Phi_{l}(t ; T)$ except the last row is $V^{(k-l-1)}(T)$. Then

$$
\begin{aligned}
& v(t)=(-1)^{k-l-1} \frac{\operatorname{det} \Phi_{l}(t-a ; b-a)}{\operatorname{det} \Psi_{l}(b-a)} ; \\
& w(t)=(-1)^{k-l-1} \frac{\operatorname{det} \Phi_{l-1}(t-a ; b-a)}{\operatorname{det} \Psi_{l}(b-a)} .
\end{aligned}
$$

This formula is useful when you can select the basis $V$ so as to make $\Psi_{l}$ close to the identity.

The constants appearing in (3.3) are

$$
\begin{aligned}
v^{(l)}(a) & =\frac{\operatorname{det} \Psi_{l+1}(b-a)}{\operatorname{det} \Psi_{l}(b-a)} ; \\
w^{(k-l)}(b) & =(-1)^{k-l-1} \frac{\operatorname{det} \Psi_{l-1}(b-a)}{\operatorname{det} \Psi_{l}(b-a)}
\end{aligned}
$$

Therefore, in terms of an arbitrary basis $v_{1}, \cdots, v_{k}$ we have the estimate

$$
\begin{aligned}
&|G(t, s)| \leqq \frac{\operatorname{det} \Phi_{i}(t-a ; b-a) \operatorname{det} \Phi_{i}(b-s ; b-a)}{\operatorname{det} \Psi_{i+1}(b-a)\left|\operatorname{det} \Psi_{i}(b-a)\right|}, \\
& \quad i=l-1, l,
\end{aligned}
$$

valid for any constant coefficient operator $K$, disconjugate on $[a, b]$.

In the case of lower order operators, $k=2,3,4$ especially, we usually try to guess $v(t), w(t)$ first, then resort to (5.4) upon failure. Therefore, we sometimes prefer

$$
G(t, s) \mid \leqq \min \left\{\frac{v(t) v(b+a-s)}{v^{(l)}(a)}, \frac{w(t) w(b+a-s)}{w^{(k-l)}(b)}\right\} .
$$

Let us now turn our attention to (3.3) The reader can fill in the details for $(3.3)_{c}$.

EXAMPLE 5.1. The operator $K v=v^{\prime \prime \prime}+v^{\prime}$ on $[0, T], T<2 \pi$. 
The $(2,1)$-problem and $(1,2)$-problem have Green's functions $G_{2,1}$ and $G_{1,2}$ satisfying (5.5), and it is easy to see that

$$
\begin{gathered}
v_{2,1}=\left[\frac{\sin t / 2}{\sin T / 2}\right]^{2}, v_{1,2}=\frac{2 \sin \left[\frac{t}{2}\right] \sin \left[\frac{T-t}{2}\right]}{\sin \left[\frac{T}{2}\right]}, \\
w_{2,1}(t)=v_{1,2}(t), w_{1,2}(t)=v_{2,1}(T-t) .
\end{gathered}
$$

Therefore,

$$
\begin{aligned}
& \left|G_{1,2}(s, t)\right|,\left|G_{2,1}(t, s)\right| \leqq \frac{2 \sin ^{2}\left[\frac{t}{2}\right] \sin ^{2}\left[\frac{T-s}{2}\right]}{\sin ^{2}\left[\frac{T}{2}\right]} \\
& \left|G_{2,1}(t, s)\right|,\left|G_{1,2}(t, s)\right| \leqq \frac{4 \sin \left[\frac{t}{2}\right] \sin \left[\frac{T-t}{2}\right] \sin \left[\frac{s}{2}\right] \sin \left[\frac{T-s}{2}\right]}{\sin ^{2}\left[\frac{T}{2}\right]}
\end{aligned}
$$

EXAMPLE 5.2. The operator $K y=y^{i v}-y$ on $[0, T], T \leqq 4.73$.

The solution $y=\sin (t)-\sinh (t)$ has three zeros at 0 and is positive for $t>0$. Therefore, the critical value of $T$ is the first positive double zero $T_{0}$ of $z=\cosh t-\cos t+C(\sinh t-\sin t)$, which is determined by the identity $\cos T_{0} \cosh T_{0}=1$. Solving numerically, $4.7300<$ $T_{0}<4.7301$.

The following inequalities follow from $(3.3)_{b}$ :

$$
\begin{gathered}
\left|G_{3,1}(t, s)\right| \leqq \frac{[\sinh (t)-\sin (t)][\sinh (T-s)-\sin (T-s)]}{2(\sinh (T)-\sin (T))}, \\
\left|G_{1,3}(t, s)\right| \leqq\left[\frac{1-\cosh T \cos T}{\sinh T-\sin T}\right] v_{2,2}(T-t) v_{2,2}(s) \\
\left|G_{2,2}(t, s)\right| \leqq\left[\frac{1-\cosh T \cos T}{\sinh T-\sin T}\right] v_{2,2}(t) v_{2,2}(T-s)
\end{gathered}
$$

where

$$
\begin{aligned}
v_{2,2}(t)= & {\left[\frac{\cosh t-\cos t}{\cosh T-\cos T}-\frac{\sinh t-\sin t}{\sinh T-\sin T}\right] } \\
& \times \frac{(\cosh T-\cos T)(\sinh T-\sin T)}{2[1-\cosh T \cos T]} .
\end{aligned}
$$

These results can be formulated for the operator $K_{a} z=z^{i v}-a^{4} z$ as well, since $z(x)=y(a x)$. The relations (3.3) are obtained from the 
identities $w_{1,3}(t)=v_{3,1}(T-t), w_{2,2}(t)=v_{2,2}(T-t), w_{3,1}(t)=v_{2,2}(t) / v_{2,2}^{\prime \prime}(0)$.

EXAMPLE 5.3. The operator $K=(D-\alpha)^{k}$ on $[a, b]$.

The Libri factorization $K v=e^{\alpha t}\left(e^{-\alpha t} v\right)^{(k)}$ is immediate from

$$
(D-\alpha) v=e^{\alpha t}\left(e^{-\alpha t} v\right)^{\prime},
$$

therefore $K$ is disconjugate on any $[a, b]$. This factorization allows us to verify by inspection that in $(3.3)_{b}$,

$$
v(t)=e^{\alpha(t-b)} \frac{(t-a)^{l}(b-t)^{k-l-1}}{(k-l-1) !(b-a)^{l}}
$$

because of what we know about $D^{k}$. Then inequality $(3.3)_{\mathrm{b}}$ is

$$
|G(t, s)| \leqq \frac{e^{\alpha(t-s)}(t-a)^{l}(b-t)^{k-l-1}(b-s)^{l}(s-a)^{k-l-1}}{l !(k-l-1) !(b-a)^{k-1}}
$$

ExAmPLE 5.4. The operator $K=\left[D^{2}-2 \alpha D+\alpha^{2}+\beta^{2}\right]^{2}$ on $[0, T]$.

The equation $K v=0$ can be replaced by $\left(D^{2}+1\right)^{2} y=0$ by virtue of the relation $v(t)=y(\beta t) e^{\alpha t}$. The largest interval of disconjugacy for $\left(D^{2}+1\right)^{2} y=0$ is $\left[0, T_{0}\right]$, where $T_{0}$ is the smallest positive root of $\tan T_{0}=T_{0}$. So $T_{0}=4.4916$, and $0<T \leqq 4.4916 \beta$.

The functions $\bar{v}_{i, j}(t)(i+j=4)$ given by

$$
\begin{aligned}
& \bar{v}_{3,1}(t)=\frac{\sin t-t \cos t}{\sin T_{0}-T_{0} \cos T_{0}}=\bar{v}_{1,3}\left(T_{0}-t\right), \\
& \bar{v}_{2,2}(t)=\frac{\left(t-T_{0}\right) \sin t \sin T_{0}+T_{0} t \sin \left(T_{0}-t\right)}{\sin ^{2} T_{0}-T_{0}^{2}}
\end{aligned}
$$

are related to the desired functions $v_{i, j}(t)(i+j=4)$ by the identity

$$
v_{i, j}(t)=\beta^{-j} \bar{v}_{i, j}(\beta t) e^{\alpha(t-T)}, \quad \mathrm{i}+j=4,
$$

where $\beta T_{0}=T$.

EXAMPLE 5.5. The operator $K=(D-\alpha)^{2}(D-\beta)^{2}$ on $[0, T]$.

The $(2,2),(3,1)$ and $(1,3)$-problems satisfy (5.5). Here,

$$
v_{2,2}(t)=-\left[\frac{\Delta_{1}}{\Delta}+\frac{\Delta_{2}}{\Delta} t+\frac{\Delta_{3}}{\Delta} e^{(\beta-\alpha) t}+\frac{\Delta_{4}}{\Delta} t e^{(\beta-\alpha) t}\right] e^{\alpha t}
$$

with

$$
\begin{aligned}
\Delta_{1} & =T\left(e^{(\beta-\alpha) T}-1\right), \quad \Delta_{3}=-\Delta_{1}, \\
\Delta_{2} & =[(\beta-\alpha) T-1] e^{(\beta-\alpha) T}+1, \quad \Delta_{4}=e^{(\beta-\alpha) T}-(\beta-\alpha) T-1, \\
\Delta & \left.=e^{2(\beta-\alpha) T}-\{[(\beta-\alpha)] T]^{2}+2\right\} e^{2(\beta-\alpha) T}+1
\end{aligned}
$$


By inspection of $D^{2}(D-\gamma)^{2}, \gamma=\beta-\alpha$, we see that

$$
\begin{aligned}
& v_{3,1}(t)=\frac{2\left[e^{\alpha t}-e^{\beta t}\right]+(\beta-\alpha) t\left[e^{\alpha t}+e^{\beta t}\right]}{(\beta-\alpha)^{3}(1+(\beta-\alpha) T) e^{(\beta-\alpha) T}}, \\
& v_{1,3}(t)=\left[e^{-\alpha(T-t)} v_{3,1}(T-t)\right] e^{\alpha t} .
\end{aligned}
$$

On the other hand,

$$
w_{3,1}(t)=\frac{v_{2,2}(t)}{v_{2,2}^{\prime \prime}(0)}, w_{1,3}(t)=v_{1,3}(T-t), w_{2,2}(t)=v_{2,2}(T-t) .
$$

6. The two-point inequality for operators $K$ with coefficients in $C[a, b]$. Throughout the preceding sections it was assumed that the coefficients of $K$ were smooth enough to define the adjoint operator $K^{*}$. It will be shown below that this smoothness assumption can be deleted, and replaced by the usual requirement that the coefficients belong only to $C[a, b]$.

LEMmA 6.1. Let $K=D^{k}+\sum_{j=0}^{k-1} p_{j}(t) D^{j}, D=d / d t$, with $p_{j} \in C[a, b]$, $0 \leqq j \leqq k-1$. If $K$ is disconjugate on $[a, b]$, then there exists $\varepsilon>0$ such that $q_{j} \in[a, b], 0 \leqq j \leqq k-1$, and $\max \left\{\left|p_{j}(t)-q_{j}(t)\right|: a \leqq\right.$ $t \leqq b\}$ less than $\varepsilon, 0 \leqq j \leqq k-1$, implies $L=D^{k}+\sum_{j=0}^{k-1} q_{j}(t) D^{j}$ is disconjugate on $[a, b]$.

Proof. Proceed indirectly, using the results in Hartman [7], p. 55. If

LEMma 6.2. Let $K=D^{k}+\sum_{j=0}^{k-1} p_{j}(t) D^{j}$ be disconjugate on $[a, b]$.

$$
K_{n}=D^{k}+\sum_{j=0}^{k-1} q_{j, n}(t) D^{j}
$$

and $q_{j, n} \rightarrow p_{j}$ as $n \rightarrow \infty$ in $C[a, b], 0 \leqq j \leqq k-1$, then the Green's functions $G_{n}(t, s)$ and $G(t, s)$ for the operators $K_{n}$ and $K$, respectively, satisfy

$$
\lim _{n \rightarrow \infty} G_{n}(t, s)=G(t, s),
$$

pointwise in $[a, b] \times[a, b]$.

Proof. Let $U_{0}(t)$ and $U_{n}(t)$ be row vector bases for $K u=0$ and $K_{n} u=0$, respectively, and denote the corresponding Wronskian matrices by $W_{0}(s)$ and $W_{n}(s)$. Suppose that $U_{0}$ and $U_{n}$ are chosen so that the representation (2.4) holds for $G$ and $G_{n}$. Since $V(s)$ is the same in each representation, it suffices to prove that $U_{n}^{(i)}(t)$ converges uniformly on $[a, b]$ to $U_{0}^{(i)}(t)$ as $n \rightarrow \infty, 0 \leqq i \leqq k-1$. This can be done using the results in Hartman [7], p. 55. The details 
are left to the reader.

To introduce notation for the next lemma, let $K$ be a disconjugate operator on $[a, b]$ and denote the basis $U$ of $\S 2$ by

$$
u_{i j}^{*}(t ; l, K) \text {. }
$$

Let $w(s ; l, K)$ be the determinant of the Wronskian matrix $W(s)$ of the basis $U$ and denote by $w_{i j}(s ; l, K)$ the minor of the element $u_{i j}^{*}(s ; l, K)$ appearing in $w(s ; l, K)$. Define

$$
\begin{gathered}
\varphi_{l}(s ; K)=\sum_{j=0}^{k-l-1} u_{1, j}^{*(l)}(a ; l, K) \cdot \frac{w_{1, j}(s ; l, K)}{w(s ; l, K)}, \\
\psi_{k-l}(s ; K)=\sum_{j=0}^{l-1} u_{0, j}^{*(k-l)}(b ; l, K) \cdot \frac{w_{0, j}(s ; l, K)}{w(s ; l, K)} .
\end{gathered}
$$

Lemma 6.3 Let $K_{n}$ and $K$ be defined as in Lemma 6.2 and assume that $q_{j, n} \rightarrow q_{j}$ as $n \rightarrow \infty$ in $C[a, b], 0 \leqq j \leqq k-1$, then $\varphi_{l}\left(s ; K_{n}\right) \rightarrow \varphi_{l}(s ; K)$ and $\psi_{k-l}\left(s ; K_{n}\right) \rightarrow \psi_{k-l}(s ; K)$ as $n \rightarrow \infty$ uniformly on $a \leqq s \leqq b$.

Proof. We argue as in Lemma 6.1 that $U_{n}^{(i)}(t) \rightarrow U_{0}^{(i)}(t)$ as $n \rightarrow$ $\infty$ uniformly on $[a, b], 0 \leqq i \leqq k-1$. It follows that the components in the formula (6.1) with $K$ replaced by $K_{n}$ converge uniformly on $[a, b]$ to the corresponding components of (6.1), as $n \rightarrow \infty$. A similar statement holds for relation (6.2). The proof is complete.

Suppose that we select $K_{n}$ to converge to $K$ in the sense of the preceding lemmas, but $K_{n}$ has coefficients of class $C^{k}$ and $K$ has coefficients that are only assumed to be continuous. By Lemma 6.1 the Green's function $G_{n}(t, s)$ exists for the operator $K_{n}$ and we can apply Theorem 3.1 to obtain the inequality

$$
\begin{array}{r}
\left|G_{n}(t, s)\right| \leqq \min \left\{\frac{1}{v_{n}^{(l)}(a)} \cdot v_{n}(t) v_{n}^{*}(s), \frac{1}{\left|w_{n}^{(k-l)}(b)\right|} \cdot w_{n}(t) w_{n}^{*}(s)\right\}, \\
n=1,2, \cdots .
\end{array}
$$

We let $n \rightarrow \infty$ in relation (6.3) and apply Lemma 6.2 and Lemma 6.3 to get the inequality

$$
|G(t, s)| \leqq \min \left\{\frac{1}{v^{(l)}(a)} \cdot v(t)\left|\varphi_{l}(s)\right|, \frac{1}{\left|w^{(k-l)}(b)\right|} \cdot w(t)\left|\psi_{k-l}(s)\right|\right\},
$$

where $\varphi_{l}(s)=\varphi_{l}(s ; K)$ and $\psi_{k-l}(s)=\psi_{k-l}(s ; K)$; indeed, the proof of Lemma 3.2 shows that $v_{n}^{*}(t)=(-1)^{k-l-1} \varphi_{l}\left(t ; K_{n}\right)$ and similarly it is easy to show that $w_{n}^{*}(t)=\left|\psi_{k-l}\left(t ; K_{n}\right)\right|$.

Inequality (6.4) does not require the existence of $K^{*}$. The right hand side of inequality (6.4) is computed directly from the basis $U$. 
7. Applications There are many immediate applications of inequality (3.3). The first and most ovbions is the theory of two-point boundary value problems for $k^{\text {th }}$ order nonlinear ordinary differential equations. Applications amount to finding conditions such that the mapping

$$
T y=\int_{a}^{b} G(t, s) f\left(s, y(s), \cdots, y^{(k-1)}(s)\right) d s+\phi(t)
$$

is a contraction mapping, or maps a closed convex set into itself, or satisfies certain fixed-point index conditions.

The second application is to intervals of uniqueness for linear differential operators. This kind of application is illustrated in the work of Ostroumov [9], and in subsequent work of Hartman [6], Willett [12], Fink [4].

The sharpness of inequality (3.3) makes it useful for error analysis. Ramifications in the theory of differential equations in Banach spaces and in the theory of functional differential equations should be clear, especially in the conversion of boundary value problems to integral equations and in the estimation of norms of inverse operators.

The requirement of disconjugacy can sometimes by checked via algebraic inequalities involving the coefficients of $K$; see Hartman [6], Ostroumov [9], Willett [12], Coppel [3], Gustafson and Bogar [2]. There is some evidence that this requirement can be dropped for self-adjoint equations, provided it is replaced by another, more complicated condition.

\section{REFERENCES}

1. P. R. Beesack, On the Green's function of an N-point boundary value problem, Pacfic J. Math., 12 (1962), 801-812.

2. G. A. Bogar and G. B. Gustafson, Effective estimates of invertibility intervals for linear multipoint boundary value problems, The University of Utah, 1974.

3. W. A. Coppel, Disconjugacy, Lecture notes in Mathematics \#220, Springer-Verlag, New York (1971).

4. A. M. Fink, Conjugate inequalities for functions and their derivatives, SIAM J. Math. Anal., 5 (1974), 399-411.

5. G. B. Gustafson, A Green's function convergence principle, with applications to computation and norm estimates, To appear, The Rocky Mountain J. Math.

6. P. Hartman, On disconjugacy criteria, Proc. Amer. Math. Soc., 24 (1970), 374-381.

7. — Ordinary Differential Equations, John Wiley and Sons, Inc., New York (1964).

8. G. Libri, Mémoire sur la résolution des équations algébriques dont les racines ont entre elles un rapport donné, et sur l'intégration des équations différentielles linéaires don les intégrales paticuliéres peuvent séxprimer les unes par les autres, J. Reine Angew. Math., 10 (1833), 167-194.

9. V. V. Ostroumov, Unique solvability of the de la Vallée Poussin problem, Differential Equations, 4 (1968), 135-139. 
10. T. L. Sherman, Properties of solutions of $N^{\text {th }}$ order linear differential equations, Pacific J. Math., 15 (1965), 1045-1060.

11. D. W. Willett, Oscillation Theory of $N^{\text {th }}$ Order Linear Differential Equations, Lecture Notes, Tne University of Utah, Salt Lake City, Utah (1973).

12. - Generalized de la Vallēe Poussin disconjugacy tests for linear differential equations, Canad. Math. Bull., 14 (1971), 419-428.

Received march 21, 1975, and in revised form June 18, 1975. The second author was partially supported by the U. S. Army under grant number DACH-04-74-G-0208.

UNIVERSITY OF UTAH 



\title{
PACIFIC JOURNAL OF MATHEMATICS
}

\section{EDITORS}

RichaRd ARENS (Managing Editor)

University of California

Los Angeles, California 90024

\section{R. A. Beaumont}

University of Washington

Seattle, Washington 98105
J. DUGUNDJI

Department of Mathematics University of Southern California Los Angeles, California 90007

D. Gilbarg and J. Milgram

Stanford University

Stanford, California 94305

\section{ASSOCIATE EDITORS}
E. F. BECKENBACH
B. H. NeUmanN
F. WOLF
K. YoshIDA

\section{SUPPORTING INSTITUTIONS}

\author{
UNIVERSITY OF BRITISH COLUMBIA \\ CALIFORNIA INSTITUTE OF TECHNOLOGY \\ UNIVERSITY OF CALIFORNIA \\ MONTANA STATE UNIVERSITY \\ UNIVERSITY OF NEVADA \\ NEW MEXICO STATE UNIVERSITY \\ OREGON STATE UNIVERSITY \\ UNIVERSITY OF OREGON \\ OSAKA UNIVERSITY
}

\author{
UNIVERSITY OF SOUTHERN CALIFORNIA \\ STANFORD UNIVERSITY \\ UNIVERSITY OF TOKYO \\ UNIVERSITY OF UTAH \\ WASHINGTON STATE UNIVERSITY \\ UNIVERSITY OF WASHINGTON \\ AMERICAN MATHEMATICAL SOCIETY
}

The Supporting Institutions listed above contribute to the cost of publication of this Journal, but they are not owners or publishers and have no responsibility for its content or policies.

Mathematical papers intended for publication in the Pacific Journal of Mathematics should be in typed form or offset-reproduced, (not dittoed), double spaced with large margins. Underline Greek letters in red, German in green, and script in blue. The first paragraph or two must be capable of being used separately as a synopsis of the entire paper. Items of the bibliography should not be cited there unless absolutely necessary, in which case they must be identified by author and Journal, rather than by item number. Manuscripts, in triplicate, may be sent to any one of the editors. Please classify according to the scheme of Math. Reviews, Index to Vol. 39. All other communications should be addressed to the managing editor, or Elaine Barth, University of California, Los Angeles, California, 90024.

The Pacific Journal of Mathematics expects the author's institution to pay page charges, and reserves the right to delay publication for nonpayment of charges in case of financial emergency.

100 reprints are provided free for each article, only if page charges have been substantially paid. Additional copies may be obtained at cost in multiples of 50 .

The Pacific Journal of Mathematics is issued monthly as of January 1966. Regular subscription rate: $\$ 72.00$ a year (6 Vols., 12 issues). Special rate: $\$ 36.00$ a year to individual members of supporting institutions.

Subscriptions, orders for back numbers, and changes of address should be sent to Pacific Journal of Mathematics, 103 Highland Boulevard, Berkeley, California, 94708.

PUBLISHED BY PACIFIC JOURNAL OF MATHEMATICS, A NON-PROFIT CORPORATION

Printed at Kokusai Bunken Insatsusha (International Academic Printing Co., Ltd.), 8-8, 3-chome, Takadanobaba, Shinjuku-ku, Tokyo 160, Japan.

Copyright (C) 1975 by Pacific Journal of Mathematics Manufactured and first issued in Japan 


\section{Pacific Journal of Mathematics}

\section{Vol. 59, No. $2 \quad$ June, 1975}

Aharon Atzmon, A moment problem for positive measures on the unit disc ........

Peter W. Bates and Grant Bernard Gustafson, Green's function inequalities for

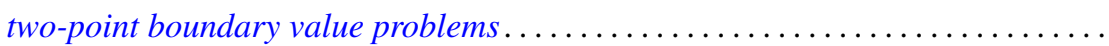

Howard Edwin Bell, Infinite subrings of infinite rings and near-rings ...........

Grahame Bennett, Victor Wayne Goodman and Charles Michael Newman, Norms of

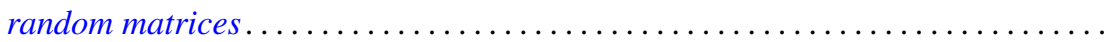

Beverly L. Brechner, Almost periodic homeomorphisms of $E^{2}$ are periodic.........

Beverly L. Brechner and R. Daniel Mauldin, Homeomorphisms of the plane ........

Jia-Arng Chao, Lusin area functions on local fields ......................

Frank Rimi DeMeyer, The Brauer group of polynomial rings ...............

M. V. Deshpande, Collectively compact sets and the ergodic theory of

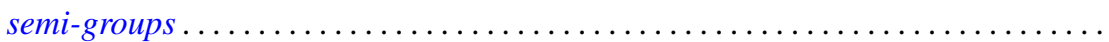

Raymond Frank Dickman and Jack Ray Porter, $\theta$-closed subsets of Hausdorff

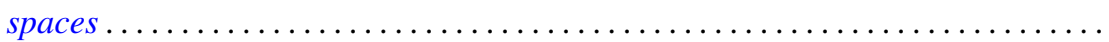

Charles P. Downey, Classification of singular integrals over a local field .......... 407

Daniel Reuven Farkas, Miscellany on Bieberbach group algebras . . . . . . . . . . . .

Peter A. Fowler, Infimum and domination principles in vector lattices . . . . . . . . . .

Barry J. Gardner, Some aspects of T-nilpotence. II: Lifting properties over

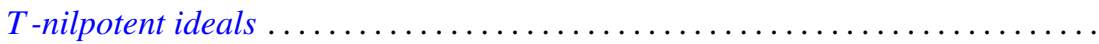

Gary Fred Gruenhage and Phillip Lee Zenor, Metrization of spaces with countable

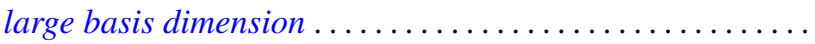

J. L. Hickman, Reducing series of ordinals...

Hugh M. Hilden, Generators for two groups related to the braid group ...

Tom (Roy Thomas Jr.) Jacob, Some matrix transformations on analytic sequence

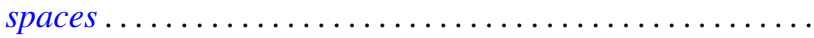

Elyahu Katz, Free products in the category of $k_{w}$-groups . .....

Tsang Hai Kuo, On conjugate Banach spaces with the Radon-Nikodým property...

Norman Eugene Liden, $K$-spaces, their antispaces and related mappings ...

Clinton M. Petty, Radon partitions in real linear spaces ........

Alan Saleski, A conditional entropy for the space of pseudo-Menger maps ....

Michael Singer, Elementary solutions of differential equations .

Eugene Spiegel and Allan Trojan, On semi-simple group algebras. I. . .

Charles Madison Stanton, Bounded analytic functions on a class of open Riemann

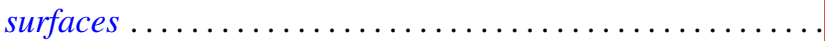

Sherman K. Stein, Transversals of Latin squares and their generalizations ....

Ivan Ernest Stux, Distribution of squarefree integers in non-linear sequences . . .

Lowell G. Sweet, On homogeneous algebras ................

Lowell G. Sweet, On doubly homogeneous algebras .......... .

Florian Vasilescu, The closed range modulus of operators ......

Arthur Anthony Yanushka, A characterization of the symplectic groups $\operatorname{PSp}(2 m, q)$

as rank 3 permutation groups... 